\title{
Vantagem Comparativa Dinâmica e Crescimento numa Economia com Dois Setores: Agrícola e Industrial*
}

\author{
Emerson Marinho ${ }^{\dagger}$, Mauricio Benegas ${ }^{\ddagger}$, Flavio Barreto ${ }^{\S}$
}

Sumário: 1. Introdução; 2. O Modelo; 3. Análise da Economia Fechada; 4. Análise da Economia Aberta; 5. Análise de Bem-Estar; 6. Formulações Alternativas; 7. Conclusões.

Palavras-chave: Crescimento endógeno; Vantagem comparativa dinâmica; Learning-by-doing.

Códigos JEL: F43; O41; Q17.

Este artigo analisa os efeitos dos ganhos de produtividade na agricultura sobre o processo de industrialização e, conseqüentemente, sobre o crescimento de longo prazo da economia. Supõe-se no modelo que a produtividade na agricultura é endógena e que a experiência acumulada na indústria exerça impacto positivo sobre a produtividade agrícola. Uma condição necessária para o declínio do emprego industrial é que, devido à vantagem comparativa na agricultura, a economia do resto do mundo apresente baixa integração agricultura/indústria Mostra-se ainda sob que condições intervenções de política podem reverter o padrão de especialização induzido pela vantagem comparativa inicial e ainda assim obter ganhos de bem estar.

This paper analyses the effects of agricultural productivity gains on the industrialization process and on the long run growth rate of the economy. The model allows endogenous gains in agricultural productivity and, besides that, it is assumed that accumulated experience in the manufacture sector exerts a positive impact on agricultural productivity. The model shown that a necessary condition to the decline of industrial employment, due to comparative advantage in agriculture, is that the economy of the rest of world has poor industry/agriculture integration. Finally, it is also shown that interventionist can reverse specialization induced by initial comparative advantages.

\footnotetext{
* Os autores agradecem os valiosos comentários dos pareceristas anônimos que foram de grande valia para tornar o texto mais claro e proveitoso. Cabe salientar que os erros remascentes são da inteira respobsabilidade dos autores. Esta pesquisa foi parcialmente financiada com resursos do CNPq.

†Professor do Curso de Pós-Graduação em Economia - CAEN/UFC. E-mail: emarinho@uf c.br

${ }^{\ddagger}$ Pesquisador do Curso de Pós-Graduação em Economia - CAEN/UFC.

$\S$ Professor do Curso de pós-Graduação em Economia - CAEN/UFC. E-mail: ataliba@uf c.br
} 


\section{INTRODUÇÃO}

O padrão de trocas internacionais tem sido reconhecido como um importante fator para a contribuição do crescimento econômico das nações. ${ }^{1}$ As altas taxas de crescimento experimentadas por países como Coréia do Sul, Hong Kong, Taiwan e Japão, ${ }^{2}$ entre o início dos anos sessenta e final dos anos oitenta, são frequentemente relacionadas às políticas de desenvolvimento "voltadas para fora", ou seja, que enfatizam setores produtivos com potencial exportador.

Apesar disso, a elaboração de modelos teóricos com o objetivo de analisar os efeitos de trocas internacionais sobre crescimento econômico foi relativamente pobre até o final dos anos oitenta e início dos anos noventa.

Nesse mesmo período os trabalhos de Romer $(1986,1990)$ e Lucas $(1988)$ passam a incorporar progresso técnico endógeno como um importante fator de crescimento econômico e, principalmente, como um meio de explicar os diferenciais de taxas de crescimento entre países.

Em Chenery (1973) a importância de uma unificação entre as teorias de crescimento econômico e padrão de trocas já era salientada. Para tanto o autor enfatiza que a noção de vantagem comparativa deveria incorporar elementos dinâmicos. Nas palavras do autor:

"A crítica principal que se faz a noção tradicional é que vantagem comparativa constitui conceito essencialmente estático que ignora uma variedade de elementos dinâmicos."'

A noção de vantagem comparativa dinâmica apresenta-se, portanto, como o principal elemento de unificação entre o padrão de trocas internacionais e a moderna teoria do crescimento endógeno.

Nos trabalhos de Grossman e Helpman (1990), Young (1991) e Matsuyama (1991, 1992), a noção de vantagem comparativa dinâmica está constantemente presente em suas análises, mas uma definição precisa desse conceito e suas consequências diretas sobre a dinâmica do padrão de trocas só aparecem em Redding (1999).

A grande maioria dos trabalhos que combina crescimento endógeno e padrão de trocas é construída com base num modelo de dois setores, um considerado tradicional (ou de baixa tecnologia) como, por exemplo, o setor agrícola e o outro de alta tecnologia, via de regra, o setor industrial.

Matsuyama (1992) considera uma economia com um setor agrícola (setor estagnante) e um outro de manufatura. Na agricultura não existe progresso técnico, enquanto na indústria o progresso técnico é endógeno via learning-by-doing. $\mathrm{O}$ autor concluiu que em economias abertas existe um link negativo entre produtividade na agricultura e emprego industrial, bem como um efeito perverso do primeiro sobre bem-estar.

No artigo de Carvalho e Barreto (2006), o progresso técnico da agricultura é endogenizado também via learning-by-doing. Os autores mostram que as conclusões de Matsuyama (1992) podem não ser sustentáveis no longo prazo devido à possibilidade de reversão de especialização induzida por vantagens comparativas.

Já Redding (1999) em seu modelo com dois setores - um de baixa tecnologia e outro de alta tecnologia, ambos com progresso técnico endógeno - mostra que, sob livre troca, pode haver redução de bem-estar intertemporal se o padrão inicial de vantagem comparativa indica que a economia não se especializa no setor no qual seu potencial de learning-by-doing é maior relativamente ao seu parceiro de troca. Neste sentido, o autor mostra que se a economia possui um potencial relativo de learning-bydoing num setor que não aquele em que se observa vantagem comparativa inicial, então intervenção na direção de reverter o padrão de especialização, induzido por vantagem comparativa, aumenta o bem estar intertemporal.

Este trabalho pretende combinar as idéias de Matsuyama (1992) e Redding (1999), construindo um modelo com dois setores: agricultura e manufatura. Em ambos os setores o progresso técnico é

\footnotetext{
${ }^{1}$ Scandizzo (1999) oferece um bom survey sobre esse assunto.

${ }^{2}$ Ver Summers e Heston. (1988).
} 
endógeno através de learning-by-doing. Além disso, supõe-se que o progresso técnico na manufatura exerce influência positiva sobre o crescimento da produtividade na agricultura.

$\mathrm{Na}$ verdade, esta última suposição pretende acomodar avanços tecnológicos do tipo land-saving (provavelmente vindo majoritariamente da indústria através de avanços nas áreas biotecnológicas e químicas) e do tipo labor-saving (em maior grau como consequência do processo de learning-by-doing do setor, mas também através da indústria devido a inovações mecânicas).

No que se segue, além dessa introdução, o artigo está distribuído da seguinte maneira: na seção 2 o modelo é formalmente descrito; na seção 3 a análise é realizada levando-se em consideração uma economia fechada. Na Seção 4, a mesma análise é desenvolvida sob a hipótese de uma economia aberta. Na seção 5, são consideradas análises de bem-estar sob hipótese de uma economia aberta. A seção 6 considera algumas formulações alternativas do modelo, explorando possíveis resultados advindos da incorporação de acumulação de capital físico e da introdução de concorrência oligopolística na indústria. Finalmente, a seção 7 apresenta as principais conclusões do trabalho bem como propõe extensões para pesquisas futuras.

\section{O MODELO}

\subsection{Tecnologia}

O modelo consiste de uma economia com dois setores: um deles, considerado de alta tecnologia, como a indústria, por exemplo, e o outro de baixa tecnologia como a agricultura. Neste sentido o produto total na economia no período $t$ é dividido entre produção agrícola, $y_{t}^{a}$, e produção na indústria, $y_{t}^{m}$. A população é constante igual a $L .^{3}$ O trabalho é o único fator de produção e a quantidade total disponível em todo período é normalizada para 1. O trabalho empregado na indústria será denotado por $l_{t}$. Assim $1-l_{t}$ é a quantidade de trabalho empregado na agricultura. Os bens industrializados são produzidos de acordo com os seguintes pressupostos tecnológicos:

$$
y_{t}^{m}=m_{t} f\left(l_{t}\right)
$$

onde, $m_{t}$, indica o nível de produtividade da mão-de-obra empregada na indústria. Supõe-se que a tecnologia na indústria satisfaça a propriedade de produtividade marginal positiva, retornos decrescentes à escala e possibilidade de inanição, ou seja, $f^{\prime}>0, f^{\prime \prime}<0$ e $f(0)=0$. A produtividade na indústria evolui de acordo com um processo de learning-by-doing, tal que:

$$
\dot{m}_{t}=\theta_{m} y_{t}^{m} \Rightarrow \dot{m}_{t}=\theta_{m} m_{t} f\left(l_{t}\right)
$$

onde, $\theta_{m}>0$, denota o potencial de learning-by-doing da economia no setor industrial.

No setor de bens agrícola, a produção satisfaz aos seguintes requisitos tecnológicos:

$$
y_{t}^{a}=a_{t} g\left(1-l_{t}\right)
$$

onde, $a_{t}$, indica o nível de produtividade da mão de obra empregada na agricultura. Da mesma forma que na manufatura, as propriedades adicionais sobre a tecnologia da agricultura são, $g^{\prime}>0, g^{\prime \prime}<0 \mathrm{e}$ $g(0)=0$. A produtividade na agricultura também evolui de acordo com um processo de learningby-doing e além disso sofre influência positiva do crescimento na produtividade da indústria. Essa suposição acomoda simultaneamente as inovações tecnológicas dos tipos land-saving e labor-saving. Por simplicidade, assume-se que esses afeitos são aditivamente separáveis. Desta forma tem-se que, $\dot{a}_{t}=\theta_{a} y_{t}^{a}+\theta y_{t}^{m}$, onde, $\theta_{a}>0$, denota o potencial de learning-by-doing do setor agrícola e, $\theta>0$, indica o grau de integração tecnológica entre a agricultura e a manufatura.

\footnotetext{
${ }^{3}$ Matsuyama (1992) mostra que o tamanho da economia não afeta os resultados obtidos.
} 
A substituição das expressões (1) e (3) na equação de mudança da produtividade agrícola $\dot{a}_{t}$, dá origem a:

$$
\dot{a}_{t}=\theta_{a} a_{t} g\left(1-l_{t}\right)+\theta m_{t} f\left(l_{t}\right)
$$

Admite-se que o efeito positivo do acúmulo de experiência no setor manufatureiro sobre a produtividade da agricultura é menos intenso do que os efeitos isolados nos respectivos setores, ${ }^{4}$ ou seja, $\theta<\min \theta_{a}, \theta_{m}$.

Por hipótese, existe pleno transbordamento de novas idéias e livre mobilidade do fator trabalho entre os dois setores da economia. Além disso, todos os mercados operam em regime de concorrência perfeita. Essas suposições são suficientes para garantir que, em equilíbrio, o valor da produtividade marginal é igual entre os setores, ou seja, a seguinte relação deve ser satisfeita:

$$
a_{t} g^{\prime}\left(1-l_{t}\right)=p_{t} m_{t} f^{\prime}\left(l_{t}\right)
$$

onde, $p_{t}$, é o preço relativo dos bens manufaturados tomando os bens agrícolas (alimentos) como numéraire.

\subsection{Preferências}

A economia é composta por agentes idênticos, cuja preferência intertemporal é expressa por:

$$
W=\int_{0}^{\infty}\left[\beta \ln \left(c_{t}^{a}-\gamma\right)+\ln c_{t}^{m}\right] e^{-\rho t} d t
$$

em que, $c_{t}^{a}$, denota o consumo per-capita de bens agrícolas (alimento) e, $c_{t}^{m}$, o consumo per-capita de bens manufaturados. Os parâmetros $\beta, \gamma$ e $\rho$, por hipótese, são todos positivos onde $\rho$ denota a taxa de desconto intertemporal. O parâmetro $\gamma$ representa o nível de subsistência no consumo de alimentos. Sua inclusão torna a utilidade do agente representativo não-homotética e consequentemente a elasticidade renda por alimentos será menor do que a unidade. Esta suposição é feita em função da lei de Enge $l^{5}$ segundo a qual o consumo de alimentos cresce absolutamente com a renda mas diminui em termos relativos. Entre os vários trabalhos que comprovam empiricamente esse resultado, podem ser destacados os de Crafts (1980) e Selvanathan e Selvanathan (2004). Echevarria (2000) utiliza o modelo de Solow para analisar as implicações da lei de Engel sobre crescimento e convergência em renda e, Steger (2000), com o mesmo propósito, utiliza um modelo do tipo Ramsey-Cass-Koopmans.

Duas hipóteses sobre consumo de subsistência serão mantidas nesse trabalho: 1) os consumidores têm renda suficiente para adquirir mais do que $\gamma$ unidades de alimentos; 2) em todo momento a agricultura é suficientemente produtiva para prover consumo de subsistência para todos os consumidores, ou seja:

$$
a_{t} g(1)>\gamma L \text {, para todo } t
$$

\section{ANÁLISE DA ECONOMIA FECHADA}

Sob a hipótese de uma economia fechada e observando que a utilidade do agente representativo é crescente no consumo de bens agrícolas e industriais a restrição orçamentária da economia deve ser

\footnotetext{
${ }^{4}$ De acordo com as especificações 2 e 4, learning-by-doing é ilimitado em ambos os setores. Para modelos com learning-by-doing limitado ver Young (1991) e Redding (1999).

${ }^{5}$ Atribuído à Ernst Engel por seu trabalho publicado em 1857.
} 
satisfeita com igualdade. Isto é, prevalece a identidade $L\left(c_{t}^{a}+p_{t} c_{t}^{m}\right)=y_{t}$, onde, $L c_{t}^{a}$, é o consumo agregado de bens agrícolas, $L c_{t}^{m}$, o consumo agregado de bens manufaturados e, $y_{t}$, é a renda agregada da economia, ou seja, $y_{t}=y_{t}^{a}+p_{t} y_{t}^{m}$. Desta forma o problema do agente representativo é dado como segue.

$$
\begin{aligned}
& \max _{\left\{c_{t}^{A}, c_{t}^{M}\right\}} \int_{0}^{\infty}\left[\beta \ln \left(c_{t}^{a}-\gamma\right)+\ln c_{t}^{m}\right] e^{-\rho t} d t \\
& \text { sujeito } a \\
& c_{t}^{a}+p_{t} c_{t}^{m}=y_{t} / L
\end{aligned}
$$

Das condições de primeira ordem do problema acima se obtêm:

$$
c_{t}^{a}=\gamma+\beta p_{t} c_{t}^{m}
$$

Denotando o consumo agregado por letra maiúscula, a expressão (8) pode ser reescrita como:

$$
C_{t}^{a}=\gamma L+\beta p_{t} C_{t}^{m}
$$

Sob o pressuposto de que a economia é fechada devem ser satisfeitas as identidades $C_{t}^{m} \equiv m_{t} f\left(l_{t}\right)$ e $C_{t}^{a} \equiv a_{t} g\left(1-l_{t}\right)$. Usando esses fatos e a condição de equilíbrio expressa pela equação (5), a equação (9) se reduz a:

$$
g\left(1-l_{t}\right)-\beta g^{\prime}\left(1-l_{t}\right) \frac{f\left(l_{t}\right)}{f^{\prime}\left(l_{t}\right)}=\frac{\gamma L}{a_{t}}
$$

A especificação do processo de learning-by-doing, de acordo com o sistema de equações diferenciais (2) e (4), faz com que os níveis de produtividade em ambos os setores sejam funções do nível de emprego na indústria, ou seja, $a_{t}=a\left(l_{t}\right)$ e $m_{t}=m\left(l_{t}\right)$. Sob o regime de autarquia, o emprego na indústria cresce com a produtividade na agricultura e a participação do emprego nesta última declina ao longo do tempo. É o que mostra a Proposição 3.1 a seguir. Para esta e demais proposições a seguir suas demonstrações exigem que a equação (10) apresente solução. ${ }^{6}$

Proposição 3.1. Numa economia fechada e sob o regime de concorrência perfeita, $l^{\prime}\left(a_{t}\right)>0$ e $d\left(1-l_{t}\right) / d t<$ 0 .

Demonstração. Observe que a equação (10) resulta das hipóteses de uma economia fechada e sob o regime de concorrência perfeita. Logo, rearranjando a equação (10), é possível obter uma relação explícita entre $a_{t}$ e $l_{t}$ tal que:

$$
a\left(l_{t}\right)=\frac{\gamma L}{g\left(1-l_{t}\right)-\beta g^{\prime}\left(1-l_{t}\right) \frac{f\left(l_{t}\right)}{f^{\prime}\left(l_{t}\right)}}
$$

Portanto diferenciando (11) com respeito a $l_{t}$ se obtêm:

$$
a^{\prime}\left(l_{t}\right)=\frac{\left(a_{t}\right)^{2}}{\gamma L}\left\{g^{\prime}\left(1-l_{t}\right)\left[1+\beta\left(1-\frac{f\left(l_{t}\right) f^{\prime \prime}\left(l_{t}\right)}{\left(f^{\prime}\left(l_{t}\right)\right)^{2}}\right)\right]-\beta g^{\prime \prime}\left(1-l_{t}\right) \frac{f\left(l_{t}\right)}{f^{\prime}\left(l_{t}\right)}\right\}
$$

Das hipóteses sobre $f\left(l_{t}\right)$ e $g\left(1-l_{t}\right)$ pode ser verificado que $a^{\prime}\left(l_{t}\right)>0$. Assim sendo, pelo teorema da função inversa, tem-se que $l_{t}=l\left(a_{t}\right) \operatorname{com} l^{\prime}\left(a_{t}\right)>0$ para qualquer $t$. Para mostrar a segunda desigualdade basta verificar que $d\left(1-l_{t}\right) / d t=-l^{\prime}\left(a_{t}\right) \dot{a}_{t}$. Como $l^{\prime}\left(a_{t}\right)>0$ o resultado segue.

${ }^{6}$ Defina $F\left(l_{t}\right)=\phi\left(l_{t}\right)-\gamma L / a\left(l_{t}\right)$. Das suposições sobre $g$ e $f$ conclui-se que a função $F$ é contínua. Além disso, $F(0)=$ $\phi(0)-\gamma L / a(0)$ com $\phi(0)=g(1)$. De acordo com a condição (7) tem-se $a(0) \phi(0)>\gamma L$, logo $F(0)>0$. Por outro lado, $F(1)=-\beta g^{\prime}(0) f(1) / f^{\prime}(1)-\gamma L / a(1)<0$. Portanto, $F$ é uma função contínua satisfazendo $F(1)<0<F(0)$. Isto posto, pelo teorema do valor Intermediário existe, $l_{t} \in(0,1)$, tal que $F\left(l_{t}\right)=0$. 
A proposição 3.1 estabelece que sob autarquia o emprego na indústria cresce com a produtividade na agricultura ao longo do tempo. Assim a visão tradicional é aqui estabelecida formalmente: os ganhos de produtividade na agricultura se refletem positivamente sobre o emprego na indústria, ou seja, a revolução "verde"é pré-condição para a revolução industrial. Este resultado tabém foi observado por Matsuyama (1992).

As evidências do declínio da participação do emprego na agricultura também foram notadas por Clark (1940), Kusnetz (1966) e Chenery e Syrquin (1975). Esses autores verificaram que a participação do emprego na agricultura declina tanto em dados seccionais quanto em séries de tempo. Portanto, o emprego na agricultura diminui ao longo do tempo e, conseqüentemente, o produto nesse setor.

Outro ponto interessante é que no modelo de uma economia fechada e sob o regime de concorrência perfeita a especialização será assintoticamente incompleta. Este resultado é formalmente estabelecido na seguinte proposição.

Proposição 3.2. Sob regime autárquico a especialização será assintoticamente incompleta, ou seja, $\lim _{t \rightarrow \infty} l_{t}=\bar{l}$ $\operatorname{com} \bar{l} \in(0,1)$.

Demonstração. Considere $\phi\left(l_{t}\right)=g\left(1-l_{t}\right)-\beta g^{\prime}\left(1-l_{t}\right) \frac{f\left(l_{t}\right)}{f^{\prime}\left(l_{t}\right)}$. Das suposições sobre $f$ e $g$ a função $\phi$ é contínua em $l_{t}$ e, assim, $\lim _{t \rightarrow \infty} \phi\left(l_{t}\right)=\phi\left(\lim _{t \rightarrow \infty} l_{t}\right)$. Desde que equação (10) possuí uma única solução $\lim _{t \rightarrow \infty} \phi\left(l_{t}\right)=\gamma L \lim _{t \rightarrow \infty} a_{t}^{-1}$. De acordo com o processo de learning-by-doing da produtividade agrícola especificado em (4), $a_{t}$ tem crescimento ilimitado e consequentemente $\lim _{t \rightarrow \infty} a_{t}^{-1}=0$. Combinando esses resultados tem-se que $\lim _{t \rightarrow \infty} \phi\left(l_{t}\right)=\phi\left(\lim _{t \rightarrow \infty} l_{t}\right)=0$, ou seja, $\lim _{t \rightarrow \infty} l_{t}=\bar{l} \operatorname{com} \bar{l} \in[0,1]$. No entanto, como, $\phi(0)>0$ e $\phi(1)<0$, tem-se que $\bar{l} \in(0,1)$.

A demonstração na nota de rodapé 6 mostra que numa economia fechada existe um nível de mão de obra empregado na indústria que satisfaz a condição de equilíbrio em (10). Além disso, esse nível de mão de obra pertence ao intervalo aberto $(0,1)$, ou seja, a especialização será incompleta para qualquer período de tempo finito. Na Proposição 3.2 por outro lado, é demonstrado que especialização também será assintoticamente incompleta. Nesse sentido, sob autarquia, sempre coexistirão ambos os setores da economia.

Por fim é possível mostrar que a participação do produto industrial é crescente com a produtividade na agricultura. De fato, denotando por $s_{m}$ a participação do produto industrial tem-se que:

$$
s_{m}=\frac{p_{t} y_{t}^{m}}{y_{t}^{a}+p_{t} y_{t}^{m}}=1 /\left[\left(\frac{m_{t} f\left(l\left(a_{t}\right)\right)}{a_{t} g\left(1-l\left(a_{t}\right)\right)}\right)^{-1}+1\right]
$$

Como $m_{t} f\left(l\left(a_{t}\right)\right) / a_{t} g\left(1-l\left(a_{t}\right)\right)$ é crescente em $l$ e $l^{\prime}\left(a_{t}\right)>0$ o resultado segue.

Os resultados obtidos sob regime autárquico, estabelecem formalmente a visão tradicional sobre a relação produtividade agrícola e industrialização, defendida dentre outros por Nurske (1953) e Rostow (1960). Além disso, esses resultados também foram estabelecidos por Matsuyama (1992) o que assegura certa robustez dos resultados encontrados. No entanto, no artigo deste último, a produtividade da agricultura é considerada exógena e constante e, portanto, o emprego na indústria também é . Neste sentido, os resultados obtidos neste artigo constituem uma generalização dos resultados de Matsuyama (1992).

Na seção seguinte a análise é estendida à economia aberta onde os resultados até aqui encontrados podem ser replicados ou não. 


\section{ANÁLISE DA ECONOMIA ABERTA}

Nesta seção são analisadas as implicações sobre o emprego e a renda considerando uma economia aberta. A economia doméstica, por hipótese, é pequena de modo que a mesma não exerce qualquer influência sobre o preço relativo dos bens manufaturados. A economia do resto do mundo apresenta as mesmas características da economia doméstica. Para efeitos de diferenciação as variáveis da economia do resto do mundo serão acrescidas de asteriscos.

Os processos de learning-by-doing na indústria e na agricultura são dados, respectivamente, por:

$$
\begin{aligned}
& m_{t}^{*}=\theta_{m}^{*} m_{t}^{*} f\left(l^{*}\right) \\
& a_{t}^{*}=\theta_{a}^{*} a_{t}^{*} g\left(1-l^{*}\right)+\theta^{*} m_{t}^{*} f\left(l^{*}\right)
\end{aligned}
$$

onde, tal como no caso da economia doméstica admite-se que $\theta^{*}<\min \theta_{a}^{*}, \theta_{m}^{*}$. Em adição, assumi-se que o emprego na indústria é constante e igual $l^{*}$.

Supõe-se ainda que exista pleno transbordamento de novas idéias e livre mobilidade do fator trabalho entre os setores da economia do resto do mundo. Desta forma, similar a economia doméstica, em equilíbrio, o valor das produtividades marginais dos setores são iguais, isto é:

$$
a_{t}^{*} g^{\prime}\left(1-l^{*}\right)=p_{t} m_{t}^{*} f^{\prime}\left(l^{*}\right)
$$

As suposições de transbordamento pleno de novas idéias e de livre mobilidade do fator de produção, não são verificados entre as economias. ${ }^{7}$ Dividindo-se a equação (16) pela equação (5) tem-se:

$$
\frac{f^{\prime}\left(l_{t}\right)}{g^{\prime}\left(1-l_{t}\right)}=\frac{a_{t}}{m_{t}} \frac{m_{t}^{*}}{a_{t}^{*}} \frac{f^{\prime}\left(l^{*}\right)}{g^{\prime}\left(1-l^{*}\right)}
$$

A Proposição 4.1 abaixo estabelece a relação entre vantagem comparativa e padrão de especialização entre a economia doméstica e a do resto do mundo para um determinado período de tempo.

Proposição 4.1. Para um dado t verifica-se

$$
l_{t}\left\{\begin{array}{c}
> \\
= \\
<
\end{array}\right\} l^{*} \Leftrightarrow \frac{a_{t}^{*}}{m_{t}^{*}}\left\{\begin{array}{l}
> \\
= \\
<
\end{array}\right\} \frac{a_{t}}{m_{t}}
$$

Demonstração. Rearranjando a equação (17) e avaliando em $t$, obtém-se:

$$
\frac{a_{t}}{m_{t}} / \frac{a_{t}^{*}}{m_{t}^{*}}=\frac{\varphi\left(l_{t}\right)}{\varphi\left(l^{*}\right)}
$$

onde $\varphi=f^{\prime} / g^{\prime}$ é uma função decrescente de $l .^{8}$ Portanto

$$
\frac{a_{t}}{m_{t}}\left\{\begin{array}{l}
> \\
= \\
<
\end{array}\right\} \frac{a_{t}^{*}}{m_{t}^{*}} \Leftrightarrow \varphi\left(l_{t}\right)\left\{\begin{array}{l}
> \\
= \\
<
\end{array}\right\} \varphi\left(l^{*}\right) \Leftrightarrow l_{t}\left\{\begin{array}{c}
< \\
= \\
>
\end{array}\right\} l^{*} .
$$

\footnotetext{
${ }^{7}$ Existe um número considerável de trabalhos que mostram empiricamente a presença de transbordamento internacional imperfeito. Ver por exemplo Coe e Helpman (1995), Branstetter (1996) e Evenson e Singh (1997). Entretanto a inclusão dessa suposição não traria ganhos significativos à análise, apenas tornando-a mais complicada.

${ }^{8}$ De fato, $\varphi^{\prime}(l)=\left[f^{\prime \prime}(l) g^{\prime}(1-l)+f^{\prime}(l) g^{\prime \prime}(1-l)\right] /\left(g^{\prime}(1-l)\right)^{2}<0$.
} 
Em palavras, a economia doméstica terá uma participação do emprego industrial maior (menor) relativamente à economia do resto do mundo se, e somente se, a mesma tiver vantagem comparativa na indústria (agricultura). A seguir, a equação (17) será utilizada para analisar a dinâmica do emprego industrial na economia doméstica. Para tanto, tomando o logaritmo natural de (17) e diferenciandoa com respeito ao tempo obtém-se a seguinte dinâmica para o emprego na indústria da economia doméstica:

$$
h\left(l_{t}\right) \dot{l}_{t}=\left(\frac{\dot{a_{t}}}{a_{t}}-\frac{\dot{m_{t}}}{m_{t}}\right)-\left(\frac{\dot{a_{t}^{*}}}{a_{t}}-\frac{\dot{m}_{t}^{*}}{m_{t}^{*}}\right)
$$

em que $h\left(l_{t}\right)=f^{\prime \prime}\left(l_{t}\right) / f^{\prime}\left(l_{t}\right)-g^{\prime \prime}\left(1-l_{t}\right) / g^{\prime}\left(1-l_{t}\right)<0$.

Segundo Redding (1999), quando se estabelece uma dinâmica de produtividade em ambos os setores da economia por meio de um processo de learning-by-doing as taxas de crescimento de produtividade passam a relacionar-se diretamente com o padrão de especialização da economia num ambiente de trocas. Com isso vantagem comparativa passa a ser endógena e faz-se necessário uma definição precisa do que seja vantagem comparativa dinâmica. Nesse trabalho será utilizada a definição dada por Redding (1999). Segundo o autor, a economia doméstica possui uma vantagem comparativa dinâmica na agricultura se, e somente se:

$$
\frac{d\left(a_{t} / m_{t}\right) / d t}{a_{t} / m_{t}}-\frac{d\left(a_{t}^{*} / m_{t}^{*}\right) / d t}{a_{t}^{*} / m_{t}^{*}}=\left(\frac{\dot{a_{t}}}{a_{t}}-\frac{\dot{m_{t}}}{m_{t}}\right)-\left(\frac{\dot{a_{t}^{*}}}{a_{t}}-\frac{\dot{m_{t}^{*}}}{m_{t}^{*}}\right)>0
$$

A equação (21) dá a formalização do conceito de vantagem comparativa dinâmica simplesmente como o análogo dinâmico da noção estática de vantagem comparativa. Com essa definição chega-se à primeira conclusão importante sobre a análise da economia aberta: desde que $h\left(l_{t}\right)<0$, de acordo com a equação (20), se a economia doméstica possuí uma vantagem comparativa dinâmica na agricultura então o emprego na indústria decresce no tempo. Esse resultado generaliza a conclusão de Matsuyama (1992) para o caso em que existe dinâmica na produtividade da agricultura. Entretanto, deve ser notado que vantagem comparativa dinâmica como definida pela equação (21), depende crucialmente do modo como a dinâmica da produtividade é especificada em ambos os setores,. Em particular, os que indicam potencial de learning-by-doing e o parâmetro que representa a integração tecnológica agricultura/indústria possuem um papel central na análise.

A fim de tornar mais precisa a análise sobre a dinâmica do emprego industrial na economia doméstica, a especificação da dinâmica da produtividade de ambos os setores será incluída. Substituindo as equações (2), (4), (14) e (15) na equação (20), obtém-se:

$$
\begin{array}{r}
h\left(l_{t}\right) \dot{l}_{t}=\left[\theta_{a} g\left(1-l_{t}\right)-\theta_{a}^{*} g\left(1-l^{*}\right)\right]+\left[\theta \frac{m_{t}}{a_{t}} f\left(l_{t}\right)-\theta^{*} \frac{m_{t}^{*}}{a_{t}^{*}} f\left(l^{*}\right)\right] \\
-\left[\theta_{m} f\left(l_{t}\right)-\theta_{m}^{*} f\left(l^{*}\right)\right]
\end{array}
$$

Observa-se que para, $\theta_{a}=\theta_{a}^{*}=\theta=\theta^{*}=0$, a equação (22) se reduz à equação (12) de Matsuyama (1992) pág. 325. Ou seja, a equação (22) generaliza o modelo desse autor incorporando elementos que endogenizam a vantagem comparativa, a saber, os parâmetros de potencial de learning-by-doing e o de integração tecnológica agricultura/indústria. A Proposição 4.2 a seguir providencia as condições necessárias e suficientes para o sinal da taxa de crescimento do emprego industrial na economia doméstica.

Proposição 4.2. Para $t \in\left[0, t^{\prime}\right)$ (onde $t \leq \infty$ ) suponha que na economia doméstica prevaleça vantagem comparativa na agricultura, isto é, $m_{t} / a_{t}<m_{t}^{*} / a_{t}^{*}$ para todo $t \in\left[0, t^{\prime}\right)$. Verificam-se as seguintes afirmações. 
a) Dado $\theta_{a}=\theta_{a}^{*}$ e $\theta_{m}=\theta_{m}^{*}$ tem-se que:

$$
\theta>\theta^{*} \text { é necessário para } \dot{l}_{t}<0
$$

b) Dado $\max \theta, \theta^{*}<\epsilon$ para $\epsilon>0$ suficientemente pequeno, tem-se que:

$$
\theta_{a}>\theta_{a}^{*} \text { e } \theta_{m}<\theta_{m}^{*} \text { é suficiente para } \dot{l}_{t}<0
$$

c) Dado $\max \theta, \theta^{*}<\epsilon$ para $\epsilon>0$ suficientemente pequeno, tem-se que:

$$
\theta_{a}<\theta_{a}^{*} \text { e } \theta_{m}>\theta_{m}^{*} \text { é necessário para } \dot{l}_{t}>0
$$

Demonstração. $\quad$ a) Dado $\theta_{a}=\theta_{a}^{*}$ e $\theta_{m}=\theta_{m}^{*}$ e recorrendo à proposição 4.1, tem-se $l_{t}<l^{*}$. Logo, das suposições sobre as funções $f$ e $g$, tem-se:

$$
\theta_{a}\left[g\left(1-l_{t}\right)-g\left(1-l^{*}\right)\right]-\theta_{m}\left[f\left(l_{t}\right)-f\left(l^{*}\right)\right]>0
$$

uma vez que $\theta_{a}>0$ e $\theta_{m}>0$. A seguir, desde que por hipótese, $m_{t} / a_{t}<m_{t}^{*} / a_{t}^{*}$, e recorredo-se novamente à Proposição 4.1, verifica-se que:

$$
\frac{m_{t}}{a_{t}} f\left(l_{t}\right)<\frac{m_{t}^{*}}{a_{t}^{*}} f\left(l^{*}\right)
$$

Portanto, afim de que, $l_{t}<0$, na equação (22) deve-se ter $\theta>\theta^{*}$, uma vez que $h\left(l_{t}\right)<0$.

b) Se o parâmetro de integração tecnológica agricultura/indústria é desprezível em ambas as economias, então, como $l_{t}<l^{*}$, segue que, $\theta_{a}>\theta_{a}^{*}$ e $\theta_{m}<\theta_{m}^{*}$, implicando $l_{t}<0$ na equação (22).

c) Recorrendo ao mesmo argumento utilizado em b), a fim de que, $l_{t}>0$, deve-se ter $\theta_{a}<\theta_{a}^{*}$ e $\theta_{m}>\theta_{m}^{*}$

De posse do resultado acima é possível estabelecer condições sob as quais o emprego na indústria cresce/decresce com a produtividade agrícola. $O$ item a) mostra que o efeito negativo da produtividade agrícola sobre o emprego na indústria requer que a integração tecnológica agricultura/indústria seja baixo na economia do resto do mundo relativamente à economia doméstica. Qual seria a intuição para este resultado? Talvez o meio mais simples de se pensar sobre isto seria inferir quais seriam as consequências se o oposto fosse observado.

Se a integração tecnológica agricultura/indústria fosse alta no resto do mundo relativamente à economia doméstica, então é razoável supor que a agricultura no resto do mundo se tornaria relativamente cada vez mais produtiva devido ao efeito de integração. Isto por sua vez levaria a economia do resto do mundo a depender cada vez menos da produção de alimentos gerada pela economia doméstica, e esta, eventualmente, veria-se obrigada a se industrializar, ${ }^{9}$ podendo assim, haver uma reversão do padrão de especialização induzida por vantagem comparativa.

Este resultado generaliza os de Matsuyama (1992) e Redding (1999). No primeiro, o autor mostra que sob a hipótese de que a produtividade na agricultura é exógena e constante em ambas as economias $\left(\theta_{a}=\theta_{a}^{*}=0\right.$ e $\left.\dot{a}=a^{*}=0\right)$, a economia doméstica se especializará assintoticamente em agricultura,

\footnotetext{
${ }^{9}$ Note que este resultado depende crucialmente da suposição de que o potencial para learning-by-doing tanto na indústria quanto
} na agricultura, sejam os mesmos para ambas as economias. 
se e somente se, a mesma possuir vantagem comparativa inicial neste setor. Entretanto, como no item a) é necessário que a integração agricultura/indústria seja relativamente baixa no resto do mundo.

Com relação ao artigo de Redding (1999), o autor estabelece que eventualmente especialização induzida por vantagem comparativa pode ser revertida em função dos potenciais de learning-by-doing das economias. No presente trabalho, viu-se que esta reversão pode ocorrer mesmo quando os potenciais de learning-by-doing são iguais em ambas as economias.

Com relação ao item b), se o efeito de integração é desprezível em ambas as economias e se o potencial de learning-by-doing na economia doméstica é relativamente maior na agricultura e menor na indústria, então vantagem comparativa inicial na agricultura é necessário e suficiente para que a economia doméstica se especialize (assintoticamente) na produção de alimentos. Para ver isso, observe que se $\theta_{a}>\theta_{a}^{*}$ e $\theta_{m}<\theta_{m}^{*}$ então, desde que o efeito de integração seja desprezível nas duas economias, tem-se:

$$
\frac{a_{0}}{m_{0}}>\frac{a_{0}^{*}}{m_{0}^{*}} \Leftrightarrow \dot{l_{t}}<0 \Rightarrow \lim _{t \rightarrow \infty} l_{t}=0
$$

Assim, a relação negativa entre a produtividade da agricultura e o emprego na indústria numa economia aberta, depende crucialmente de que os potenciais de learning-by-doing em ambas as economias estejam alinhados com a vantagem comparativa observada inicialmente. Ou seja, a agricultura na economia doméstica deve ser relativamente mais produtiva do que a agricultura no resto do mundo e a indústria relativamente menos produtiva.

Finalmente, o item c) revela que para que o emprego industrial cresça na economia doméstica sob vantagem comparativa na agricultura, é necessário que os potenciais de learning-by-doing contrariem a direção da vantagem comparativa. Isto é, a economia doméstica deve ter potencial de learning-bydoing relativamente maior na indústria e menor na agricultura. Este resultado simplesmente reverte $o$ raciocínio subjascente ao empregado na análise do item b).

Existe, de curso, uma importante implicação neste caso: economias em desenvolvimento podem enfrentar um trade-off entre especializar-se de acordo com o padrão existente de vantagem comparativa (neste caso na produção de alimentos) ou voltar-se para setores nos quais não possuem vantagem comparativa observada, mas podendo entretanto adquiri-la no futuro. Isso se daria como resultado do potencial de crescimento da produtividade devido ao processo de learning-by-doing.

Todas essas conclusões, além de generalizar resultados obtidos em trabalhos anteriores, também sugerem várias implicações de políticas voltadas para o desenvolvimento de economias menos desenvolvidas, onde, via de regra, o padrão de vantagem comparativa induz à especialização na agricultura. Se os potenciais de learning-by-doing nessas economias sugerem uma indústria produtiva, então intervenção estratégica na direção de inseri-las em atividades mesmo sem vantagens comparativas poderia fazer com que essa economia viesse a tê-las no futuro.

Reeding (1999) aponta como exemplo desse caso uma experiência interessante sobre a indústria siderúrgica Coreana. $\mathrm{O}$ autor revela que um estudo do Banco Mundial nos anos sessenta, apontava que qualquer consideração sobre a estruturação desse setor na Coréia figurava-se como "...uma proposição imatura e sem factibilidade econômica...", ou seja, não havia vantagem comparativa observada naquele setor. Entretanto o governo Coreano fundou, em 1973, a Pohang Iron and Steel Company (POSCO). Surpreendentemente, em 1988 a POSCO tornou-se a $11^{a}$ maior companhia siderúrgica do mundo, operando com 80 plantas individuais e com custos unitários que eram cerca de dois terços dos observados na indústria siderúrgica americana.

Assim se observa que quando a POSCO foi criada a Coréia não tinha qualquer vantagem naquele setor. Entretanto, seu potencial para ganhos em produtividade tornou possível não só a estruturação do setor como também passou a ser um país com indiscutível vantagem comparativa nesta atividade.

A seção seguinte é dedicada a analisar os impactos das vantagens comparativas sobre o bem-estar da economia doméstica relativamente à economia do resto do mundo. 


\section{ANÁLISE DE BEM-ESTAR}

Nesta seção pretende-se inferir o impacto das vantagens comparativas sobre o nível de bem estar no caso de uma economia aberta. Por simplicidade supõe-se a inexistência de um mercado internacional de capitais. Assim, denote por, $E_{t}=L\left(c_{t}^{a}+p_{t} c_{t}^{m}\right)$, o dispêndio agregado da economia doméstica cuja renda é dada por $y_{t}=a_{t} g\left(1-l_{t}\right)+p_{t} m_{t} f\left(l_{t}\right)$. Usando a equação (5) nesta última expressão, a renda da economia doméstica pode ser escrita como:

$$
y_{t}=a_{t}\left[g\left(1-l_{t}\right)+g^{\prime}\left(1-l_{t}\right) \frac{f\left(l_{t}\right)}{f^{\prime}\left(l_{t}\right)}\right]
$$

O problema do consumidor na economia doméstica é escolher seu consumo de bens agrícolas e de modo a maximizar sua utilidade intertemporal sujeito à restrição de dispêndio agregado da economia. Especificamente $o$ agente representativo resolve:

$$
\begin{aligned}
& \max _{c_{t}^{A}, c_{t}^{M}} \int_{0}^{\infty}\left[\beta \ln \left(c_{t}^{a}-\gamma\right)+\ln c_{t}^{m}\right] e^{-\rho t} d t \\
& \text { sujeito } a \\
& \qquad c_{t}^{a}+p_{t} c_{t}^{m}=E_{t} / L
\end{aligned}
$$

Das condições de primeira ordem do problema de maximização em (25), tem-se que os níveis de consumo per-capita de bens agrícola e manufaturado são dados, respectivamente, por:

$$
\begin{gathered}
c_{t}^{a}=\frac{\beta p_{t} E_{t}}{(1+\beta) L}+\frac{\gamma}{(1+\beta)} \\
c_{t}^{m}=\frac{E_{t}}{(1+\beta) L}-\frac{\gamma}{(1+\beta) p_{t}}
\end{gathered}
$$

Substituindo-se as equações (26) e (27) na função objetivo de (25) obtém-se a utilidade indireta do agente representativo. Denotando-a por $W_{H}$ tem-se:

$$
W_{H}=(1+\beta) \int_{0}^{\infty} \ln \left(\frac{E_{t}}{L}-\gamma\right) e^{-\rho t} d t+\Omega
$$

em que $\Omega=\beta \ln \left(\frac{\beta}{1+\beta}\right)+\ln \left(\frac{1}{1+\beta}\right)-\int_{0}^{\infty} \ln \left(p_{t}\right) e^{-\rho t} d t$. Sob a hipótese de que inexiste mercado de capitais internacional, a renda nacional deve ser igual ao dispêndio nacional para todo $t$, ou seja, $E_{t}=y_{t}$. Usando este fato e a condição (24) na equação (28), obtém-se:

$$
W_{H}=(1+\beta) \int_{0}^{\infty} \ln \left(\frac{a_{t} \psi\left(l_{t}\right)}{L}-\gamma\right) e^{-\rho t} d t+\Omega
$$

onde $\psi\left(l_{t}\right)=g\left(1-l_{t}\right)+g^{\prime}\left(1-l_{t}\right) \frac{f\left(l_{t}\right)}{f^{\prime}\left(l_{t}\right)}$ e, portanto, $\psi^{\prime}\left(l_{t}\right)>0 .^{10}$

Denotando por $W_{R W}$ a utilidade indireta do agente representativo do resto do mundo, os mesmos argumentos acima podem ser utilizados para verificar que:

$$
W_{R W}=(1+\beta) \int_{0}^{\infty} \ln \left(\frac{a_{t}^{*} \psi\left(l^{*}\right)}{L^{*}}-\gamma\right) e^{-\rho t} d t+\Omega
$$

$\overline{{ }^{10} \text { De fato } \psi^{\prime}\left(l_{t}\right)=-\left[\frac{g^{\prime}\left(1-l_{t}\right) f\left(l_{t}\right) f^{\prime \prime}\left(l_{t}\right)}{\left(f\left(l_{t}\right)\right)^{2}}+\frac{g^{\prime \prime}\left(1-f\left(l_{t}\right)\right) f\left(l_{t}\right)}{f^{\prime}\left(l_{t}\right)}\right]>0 .}$ 
de forma que $L^{*}$ denota a população do resto do mundo. Para comparar os níveis de bem estar das economias a vantagem comparativa será relacionada com o sinal da diferença $\left[W_{R W}-W_{H}\right]$. Para tanto, usando (29) e (30) tem-se:

$$
W_{H}-W_{R W}=(1+\beta) \int_{0}^{\infty} \ln \left(\frac{a_{t} \psi\left(l_{t}\right)-\gamma L}{a_{t}^{*} \psi\left(l^{*}\right)-\gamma L^{*}}\right) e^{-\rho t} d t+\ln \left(\frac{L^{*}}{L}\right)
$$

Suponha que por todo o horizonte de planejamento prevaleça vantagem comparativa na agricultura para a economia doméstica, ou seja, $a_{t} / m_{t}>a_{t}^{*} / m_{t}^{*}$ para todo $t$. Então, pela Proposição 4.1 tem-se que $l_{t}<l^{*}$ e, portanto, $\psi\left(l_{t}\right)<\psi\left(l^{*}\right)$ para todo $t$. Logo o efeito (direto) sobre o nível de emprego industrial na economia doméstica contribui para reduzir o seu bem-estar relativamente à economia do resto do mundo.

Entretanto esse efeito pode ser compensado pela presença dos níveis de produtividade da agricultura em cada economia. Com efeito, em função do processo de learning-by-doing o nível de produtividade da agricultura em cada economia depende dos parâmetros que indicam potencial de apredizagem (efeito próprio) e integração tecnológica agricultura/indústria (efeito cruzado). Logo o sinal da equação (31) é indeterminado dependendo das suposições sobre a magnitude relativa de tais parâmetros. Por exemplo, satisfeita a condição suficiente $\theta_{a}>\theta_{a}^{*}$ e $\theta_{m}<\theta_{m}^{*}$ dado $\max \theta, \theta^{*}<\epsilon$ para $\epsilon>0$ suficientemente pequeno, tem-se que a produtividade da agricultura na economia doméstica cresce mais rapidamente do que a produtividade agrícola do resto do mundo.

Pela Proposição $4.2, l_{t}<0$, o que faz com que $l_{t} \rightarrow 0$ quando $t \rightarrow \infty \operatorname{com} \psi(0)=g(1)<\infty$. Por outro lado, pelo processo de learning-by-doing para a produtividade agrícola (e supondo que o efeito de integração tecnológica é desprezível) $a_{t}$ tem crescimento ilimitado. Assim sendo, supondo que a taxa de desconto intertemporal é suficientemente baixa concluí-se que $W_{H}-W_{R W}>0$. Ou seja, a economia doméstica embora especializando-se (assintoticamente) no setor estagnante teria padrão de bem estar superior ao da economia do resto do mundo.

\section{FORMULAÇÕES ALTERNATIVAS}

Esta seção tem por objetivo apresentar possíveis resultados decorrentes das modificações de algumas das hipóteses utilizadas no modelo inicial. Inicialmente especula-se o que aconteceria se o modelo incorporasse acumulação de capital físico como fator específico da indústria. Um exercício semelhante é desenvolvido com intenção de entender possíveis consequências de se abandonar a hipótese de concorrência perfeita admitindo que a indústria opere sob condições de oligopólio. Deve-se salientar, no entanto que as análises apresentadas não são conclusivas pois os efeitos definitivos só podem ser obtidos através da resolução do modelo incorporando tais modificações.

\subsection{Capital Físico Como Fator Específico}

Inicialmente suponha que o capital fisico é dado em ambas as economias e que o mesmo é fator específico da indústria. Qual seria a implicação de uma expansão exógena do capital na economia doméstica? Se a economia doméstica é exportadora de bens agrícolas (ou seja, possui vantagem comparativa inicial nesse setor), uma expansão do capital expande a produção de bens industriais conduzindo a um aumento temporário na demanda por mão de obra nesse setor. Assim nas condições da Proposição 4.2(b) o aumento do estoque de capital da economia doméstica poderia reforçar a reversão de especialização a favor da indústria.

No cenário acima pode haver também uma melhora dos termos troca em favor da economia doméstica (redução do preço relativo de bens industriais) em vista de uma redução das importações do bem 
industrial (reduzindo a demanda relativa e aumentando a oferta relativa desse bem) o que pode incentivar por outro lado a expansão da produção de bens agrícolas.

Outro ponto que deve ser destacado é que o aumento do capital físico aumentaria a produtividade em ambos os setores, na indústria através do processo de learning-by-doing e na agricultura através da integração tecnológica entre os setores. Mas esse aumento seria proporcionalmente maior na indústria em função da hipótese de que o efeito cruzado é menos intenso do que os efeitos próprios. Recorrendo novamente à Proposição 4(b) esse efeito poderia reforçar a reversão de especialização em favor da indústria.

As reflexões acima sugerem que uma política de subsídios ao capital físico poderia ser efetiva no processo de industrialização de uma economia com vantagem comparativa na agricultura.

A introdução de uma dinâmica de acumulação de capital não teria impacto significativo na economia fechada. Os resultados de coexistência dos setores e do impacto positivo do aumento da produtividade agrícola sobre industrialização se manteriam. Mas deve ser observado que haveria um efeito imediato sobre a produtividade de ambos os setores devido aos efeitos de learning-by-doing e de integração.

$\mathrm{Na}$ economia aberta, por outro lado, os impactos podem se consideráveis. Imagine inicialmente que existe dinâmica de capital na economia doméstica, mas que o estoque de capital é dado na economia do resto do mundo. Numa situação em que o mercado de capitais internacional é perfeito e que a economia doméstica é pequena, as remunerações do capital físico nesta última seriam dadas pela taxa de juros vigente na economia do resto do mundo. Portanto, a princípio, a expansão do capital físico na economia doméstica estaria limitada pelo crescimento da economia mundial, mesmo que a primeira seja exportadora de bens agrícolas (em função da vantagem comparativa inicial nesse setor), o que sugere uma possível limitação sobre a efetividade dos subsídios ao capital físico como instrumento de política industrial.

\subsection{Oligopólio na Indústria}

De acordo com Helpman e Krugman (1986), vantagem comparativa é robusta em relação a estrutura de mercado. Supondo que a economia é aberta, a economia doméstica possui vantagem comparativa inicial e o bem manufaturado é comercializado num ambiente de concorrência oligopolística. Ou seja, indústria local e indústria do resto do mundo disputam o mercado da economia doméstica através de decisão de oligopólio. Neste caso o padrão tecnológico é crucial no momento de decidir a melhor resposta.

Ruffin (2003) corrobora a robustez de vantagem comparativa, mas argumenta que existe um threshold de competição em relação ao qual o volume e os benefícios de trocas podem mudar. Quando competição excede o nível de threshold o volume e padrão de trocas segue a lógica Ricardiana. Quando a competição está abaixo do nível de threshold então o padrão de trocas será em geral dado pela lógica Ricardiana, mas com um volume de comércio menor. Essa distinção no volume é a razão pela qual os ganhos da troca serão diferentes em relação ao nível de competição threshold.

Na comparação de bem estar feita entre as economias não haveria modificação na análise, pois a interferência do nível de competição nos ganhos de troca se daria via preço que desaparece na análise (mantendo-se o pressuposto de que a economia doméstica é pequena). A incorporação de formação de preço no oligopólio seria relevante apenas para o caso de uma economia de grande porte, o que retira da economia do resto do mundo o status de benchmark.

\section{CONCLUSÕES}

Este artigo formaliza um modelo de crescimento endógeno em que o modelo desenvolvido por Matsuyama (1992) é extendido de modo a permitir que haja progresso técnico endógeno na agricultura, bem como uma integração tecnológica entre este setor e a manufatura. 
A incorporação das especificações sobre a dinâmica da produtividade nos setores agrícola e industrial, de acordo a definição de Redding (1999), mostraram ter implicações significativas sobre a noção tradicional (estática) de vantagens comparativas.

Sob a hipótese de regime autárquico a visão tradicional sobre a relação produtividade agrícola e industrialização é formalmente estabelecida, pois se verifiica que a participação do emprego e da renda do setor industrial guarda relação positiva com a produtividade agrícola. Ademais, pode-se concluir que esse resultado é robusto uma vez que o mesmo é obtido por Matsuyama (1992) num modelo em que produtividade da agricultura é dada exogenamente.

Na análise de uma economia fechada a especialização será incompleta mesmo assintoticamente, resultado que depende em grande parte da especificação da utilidade intertemporal que acomoda a lei de Engel.

As principais diferenças entre o modelo aqui proposto e o trabalho de Matsuyama (1992) surgem na análise da economia aberta. Nesse contexto a conclusão de que existe um link negativo entre vantagem comparativa na agricultura e indústria não é de forma nenhuma direta como conclui aquele autor.

Existem várias condições subjascentes a esse resultado. A primeira delas refere-se diretamente à definição de vantagem comparativa dinâmica. Assim, para que o emprego industrial na economia doméstica decresça no tempo é suficiente que esta possua vantagem comparativa dinâmica na agricultura.

Isto posto, resta a questão: quais são os determinantes da evolução da vantagem comparativa na economia doméstica? Mostrou-se que, se os potenciais de learnig-by-doing na agricultura e na indústria são iguais entre a economia doméstica e o resto do mundo, então uma integração tecnológica relativamente baixa na economia do resto do mundo é condição necessária para que o emprego na indústria doméstica seja decrescente em função de vantagem comparativa na agricultura. De outro modo, a economia do resto do mundo dependeria cada vez menos da produção agrícola gerada pela economia doméstica fazendo com que esta fosse obrigada a se industrializar.

Finalmente, sob as hipóteses de que a integração tecnológica seja desprezível em ambas as economias e a doméstica possua vantagem comparativa inicial na agricultura, a condição necessária para que haja reversão de especialização induzida por vantagem comparativa é que o potencial de learning-bydoing nesta economia seja relativamente maior na indústria e menor na agricultura. Isto sugere que economias em desenvolvimento com vantagem comparativa inicial na agricultura, podem, no longo prazo, se especializar no setor industrial. Como um exemplo da evidência desse processo de reversão, cita-se a experiência exitosa da indústria siderúrgica coreana.

Reeding (1999) relata que um estudo do Banco Mundial nos anos sessenta, apontava que qualquer consideração sobre a estruturação desse setor na Coréia figurava-se como "...uma proposição imatura e sem factibilidade econômica...", ou seja, não havia vantagem comparativa observada naquele setor. Entretanto o governo Coreano fundou, em 1973, a Pohang Iron and Steel Company (POSCO). Surpreendentemente, em 1988 a POSCO tornou-se a $11^{a}$ maior companhia siderúrgica do mundo, operando com 80 plantas individuais e com custos unitários que eram cerca de dois terços dos observados na indústria siderúrgica americana.

Por fim uma análise relacionando vantagem comparativa e bem-estar foi conduzida mostrando que no caso de especialização induzida por vantagem comparativa a economia doméstica terá nível de bemestar maior que a economia do resto do mundo.

O trabalho faz várias suposições simplificadoras as quais abrem possibilidades de extensões do original. A primeira delas é a ausência de acumulação de capital físico. ${ }^{11}$ A sua inclusão no modelo induziria a uma otimização dinâmica de fato e uma análise mais completa, além de relaxar a hipótese de ausência

\footnotetext{
${ }^{11}$ No trabalho de Wong e Yip (1999), um modelo semelhante ao aqui apresentado, incluí acumulação de capital físico. Entretanto duas outras suposições enfraquecem a estrutura do modelo: 1) como em Matsuyama (1992) a produtividade da agricultura é dada exogenamente; 2) as tecnologias exibem retornos constantes de escala.
} 
de um mercado internacional de capítais. Como foi observado na Seção 6, uma série de novos resultados poderia emergir.

Uma última sugestão de extensão seria a inclusão de um setor produzindo bens non-tradeable e verificar o impacto desta suposição sobre o processo de industrialização ${ }^{12}$.

\section{Referências Bibliográficas}

Boldrin, M. \& Scheinkman, J. A. (1988). Learning-By-Doing, International Trade and Growth: A Note. Addison-Wesley, Reading, MA. SFI Studies in the Science of Complexity.

Branstetter, L. (1996). Are knowledge spillovers international or intranational in scope? Microeconometric evidence from the Japan and the united states. NBER Working Papers 5800, National Bureau of Economic Research, Inc. Available at http://ideas.repec.org/p/nbr/nberwo/5800 .html.

Carvalho, R. M. \& Barreto, F. A. F. D. (2006). Learning-by-doing, produtividade agrícola e crescimento econômico. Revista Brasileira de Economia, 60(1). Available at http://ideas.repec.org/a/fgv/ epgrbe/v60n1a1.html.

Chenery, H. (1973). Vantagem comparativa e medidas de política para o desenvolvimento. In Savasini, J., Malan, P., \& (org.), W. B., editors, Economia Internacional, Série Anpec de Leituras de Economia. Ed.Saraiva.

Chenery, H. \& Syrquin, M. (1975). Patterns of Development, 1950-1970. Oxford University Press.

Clark, C. (1940). The Conditions of Economic Progress. MacMillan \& Co. London.

Coe, D. \& Helpman, E. (1995). International R\&D Spillovers. European Economic Review, 39:859-887.

Crafts, N. F. R. (1980). Income elasticities of demand and the release of labor by agriculture during the British industrial revolution: a further appraisal. European Economic Review, 9:153-168.

Echevarria, C. (2000). Non-homothetic preferences and growth. Journal of International Trade $\mathcal{E}$ Economic Development, 9(2):151-171. Available at http://ideas.repec.org/a/taf/jitecd/ v9y2000i2p151-171.html.

Eswaran, M. \& Kotwal, A. (2002). The role of the service sector in the process of industrialization. Journal of Development Economics, 68(2):401-420. Available at http://ideas.repec .org/a/eee/ deveco/v68y2002i2p401-420.html.

Evenson, R. \& Singh, L. (1997). Economic growth, international technological spillovers and public policy: Theory and empirical evidence from Asia. Papers 777, Yale - Economic Growth Center. Available at http://ideas.repec.org/p/fth/yalegr/777.html.

Grossman, G. M. \& Helpman, E. (1990). Comparative advantage and long-run growth. American Economic Review, 80(4):796-815. Available at http://ideas.repec.org/a/aea/aecrev/ v80y1990i4p796-815.html.

Helpman, E. \& Krugman, P. (1986). Foreing Trade and Market Struture. MIT Press, Cambridge, MA.

Kuznets, S. (1966). Modern Economic Growth. Yale University Press, New Haven, CT.

\footnotetext{
${ }^{12}$ Eswaran e Kotwal (2002) apresenta um modelo de economia aberta com o setor de serviços, mas sem progressso técnico endógeno na indústria e sem o setor agrícola.
} 
Lucas, R. J. (1988). On the mechanics of economic development. Journal of Monetary Economics, 22(1):342. Available at http://ideas.repec.org/a/eee/moneco/v22y1988i1p3-42.html.

Matsuyama, K. (1991). Increasing returns, industrialization, and indeterminacy of equilibrium. The Quarterly Journal of Economics, 106(2):617-50. Available at http://ideas.repec .org/a/tpr/qjecon/ v106y1991i2p617-50.html.

Matsuyama, K. (1992). Agricultural productivity, comparative advantage, and economic growth. Journal of Economic Theory, 58(2):317-334. Available at http://ideas.repec.org/a/eee/jetheo/ v58y1992i2p317-334.html.

Nurske, R. (1953). Problems of Capital Formation in Underdeveloped Countries. Oxford University Press, New York.

Redding, S. (1999). Dynamic comparative advantage and the welfare effects of trade. Oxford Economic Papers, 51(1):15-39. Available at http://ideas.repec .org/a/oup/oxecpp/v51y1999i1p15-39. html.

Romer, P. M. (1986). Increasing returns and long-run growth. Journal of Political Economy, 94(5):1002-37. Available at http://ideas.repec.org/a/ucp/jpolec/v94y1986i5p1002-37.html.

Romer, P. M. (1990). Endogenous technological change. Journal of Political Economy, 98(5):S71-102. Available at http://ideas.repec.org/a/ucp/jpolec/v98y1990i5ps71-102.html.

Rostow, W. W. (1960). The Stages of Economic Growth: A Non-Communist Manifesto. Cambridge University Press, UK.

Ruffin, R. J. (2003). Oligopoly and trade: what, how much, and for whom? Journal of International Economics, 60(2):315-335. Available at http://ideas.repec.org/a/eee/inecon/v60y2003i2p315-335.html.

Scandizzo, P. L. (1999). Growth, trade and factor endowment: A survey. Labour, 13(2):509-547. Available at http://ideas.repec.org/a/bla/labour/v13y1999i2p509-547.html.

Selvanathan, S. \& Selvanathan, E. A. (2004). Empirical regularities in South African consumption patterns. Applied Economics, 36(20):2327-2333. Available at http://ideas.repec.org/a/taf/applec/v36y2004i20p2327-2333.html.

Steger, T. M. (2000). Economic growth with subsistence consumption. Journal of Development Economics, 62(2):343-361. Available at http://ideas.repec.org/a/eee/deveco/v62y2000i2p343-361.html.

Summers, R. \& Heston., A. (1988). A new set of interational comparations of real product and price: estimates of 130 countries. The Review of Income and Wealth, 34:1-25.

Wong, K.-y. \& Yip, C. K. (1999). Industrialization, economic growth, and international trade. Review of International Economics, 7(3):522-40. Available at http://ideas.repec.org/a/bla/reviec/v7y1999i3p52240.html.

Young, A. (1991). Learning-by-doing and the dynamic effects of international trade. Quarterly Journal of Economics, 106:369-405. 